\title{
ANDES

www.scielo.cl

\section{Duración de la lactancia materna, alimentación combinada y riesgo para la salud en jóvenes costarricenses}

\author{
Breastfeeding duration, mixed feeding and health risk in Costa Rican \\ children and adolescents
}

\author{
Núñez-Rivas HPa, Holst-Schumacher I ${ }^{\mathrm{b}}$, Roselló-Araya $\mathrm{M}^{\mathrm{a}}$, Campos-Saborío Nc, Guzmán-Padilla ${ }^{\mathrm{a}}$
}

alnstituto Costarricense de Investigación y Enseñanza en Nutrición y Salud (INCIENSA). Cartago, Costa Rica.

bFacultad de Microbiología, Universidad de Costa Rica y Centro de Investigación en Hematología y Trastornos Relacionados. San José. Costa Rica. Universidad Estatal a Distancia de Costa Rica. San José. Costa Rica.

Recibido: 22 de enero de 2021; Aceptado: 10 de julio de 2021

¿Qué se sabe del tema que trata este estudio?

No existe consenso claro entre la duración de la lactancia materna, la alimentación infantil y la obesidad en la infancia posterior. La mayor parte de los estudios se han concentrado en estudiar esta relación en niños menores a 6 años.

\begin{abstract}
¿Qué aporta este estudio a lo ya conocido?
Se evidenció la protección de la lactancia materna ante la obesidad en una población joven de Costa Rica; los alimentados sólo con fórmula presentaron mayor riesgo de ser obesos. Se debe asesorar sobre alimentación complementaria y saludable y actividad física durante las primeras etapas de la vida.
\end{abstract}

\section{Resumen}

Objetivo: examinar la asociación entre la duración de la lactancia materna y la alimentación mixta con la obesidad, riesgo cardiometabólico, grasa corporal, conductas de riesgo dietéticas y de actividad física en niños y adolescentes. Sujetos y Método: estudio transversal en 1467 estudiantes de Costa Rica. Se aplicó una entrevista a padres e hijos para recopilar información socioeconómica, escolaridad, conductas de riesgo de la alimentación y actividad física de los jóvenes, duración de la lactancia materna y alimentación mixta. Se evaluó el índice de masa corporal, la circunferencia abdominal y la grasa corporal mediante análisis de bioimpedancia. El riesgo cardiometabólico se midió con el indicador cintura/talla (WHtR $\geq 0,5)$. Las mediciones fueron realizadas por personal estandarizado. Se aplicó estadística descriptiva y regresión logística. Resultados: la edad de los sujetos fue $11,4 \pm 2,6$ años; $50,9 \%$ hombres; $55,5 \%$ clase media; $60 \%$ sedentarios y $16 \%$ obesos. El $20 \%$ fue amamantado sin introducción de fórmula hasta los 6 meses y $13 \%$ nunca fue amamantado; más del $60 \%$ fue amamantado por $\geq 6$ meses. El porcentaje de obesidad de los que recibieron leche materna, sola o en combinación con fórmula, durante $\geq 6$ meses fue menor que los que fue-
Palabras clave:

Lactancia Materna; Obesidad;

Fórmula;

Alimentación Mixta;

Conductas de Riesgo
Correspondencia:

Marlene Roselló-Araya

mrosello@inciensa.sa.cr
Editado por:

Gerardo Weisstaub 
ron alimentados solo con fórmula $(60,8$ vs 39,2; p < 0,005). Los niños con riesgo cardiometabólico, dieta no saludable, sedentarismo y que fueron alimentados sólo con fórmula, presentaron mayor riesgo de ser obesos (OR = 18,8, IC 95\% 13,2-26,0). Conclusiones: estos resultados son consistentes con otros estudios y refuerzan la protección evidente que tiene la lactancia materna frente al desarrollo de la obesidad.

\begin{abstract}
Objective: to analyze the associations between breastfeeding duration and mixed feeding (breast milk and formula) with obesity, cardiometabolic risk (WHtR), body fat, and dietary and physical activity risk behaviors in children and adolescents. Subjects and Method: cross-sectional study carried out with 1,467 students in Costa Rica. An interview was conducted with parents and children to gather information on socioeconomic status, education, dietary and physical activity risk behaviors of the youth, including breastfeeding duration and mixed feeding. Body mass index, waist circumference, and body fat were assessed by bioelectrical impedance analysis and cardiometabolic risk was measured with the waist-to-height ratio $(\mathrm{WHtR} \geq 0.5)$ by trained personnel. Descriptive statistics and logistic regression models were applied. Results: the average age was $11.4 \pm 2.6$ years and $50.9 \%$ were male. $55.5 \%$ of the population was middle class; $60 \%$ were sedentary, and $16 \%$ presented obesity. Around 20\% were breastfed without the introduction of formula before 6 months, 13\% were never breastfed, and more than $60 \%$ were breastfed for $\geq 6$ months. Those children who were fed only with breast milk or in combination with formula for $\geq 6$ months presented a lower percentage of obesity than those who received formula feeding only $(60.8$ vs $39.2 ; \mathrm{p}<0.005)$. Children with cardiometabolic risk (WHtR $\geq 0.50$ ), unhealthy diet, sedentary lifestyle, and who were fed only with formula are at higher risk of developing obesity $(\mathrm{OR}=18.8,95 \% \mathrm{CI} 13.2-26.0)$. Conclusions: these results are consistent with other studies and reinforce the evident protection of breastfeeding against the development of obesity.
\end{abstract}

Keywords:

Breastfeeding;

Obesity;

Formula;

Mixed Feeding;

Risk Behaviors

\section{Introducción}

La lactancia materna (LM) está relacionada con una mejor condición de salud y es uno de los factores protectores que se ha estudiado rigurosamente en relación con la prevención de la obesidad infantil y las enfermedades crónicas no transmisibles (ECNT). Asimismo, los beneficios físicos y psicológicos de la LM para los lactantes y las madres son numerosos y se han descrito ampliamente ${ }^{1,2}$; se aceptan los beneficios para la salud de la LM sobre el uso de la fórmula, sin embargo, no existe un consenso claro en la literatura sobre la relación entre la duración de la lactancia materna, la alimentación infantil y la obesidad en la infancia posterior ${ }^{3}$ y la mayor parte de los estudios se han concentrado en estudiar esta relación en niños menores a 6 años ${ }^{4}$.

La Organización Mundial de la Salud señala que se debe iniciar la LM dentro de la primera hora después del nacimiento y continuarla exclusivamente hasta al menos los seis meses de edad; momento en el que se pueden introducir gradualmente los alimentos complementarios. Además, se recomienda continuar con la lactancia hasta que el niño tenga dos años o más. Sin embargo, pocos estudios han llegado a conclusiones contundentes relacionadas con la duración de la LM y la alimentación mixta (leche materna y fórmula) y su asociación con la obesidad, el riesgo cardiometabólico y la grasa corporal (adiposidad infantil) ${ }^{3,5,6}$, y los existentes se han enfocado en el riesgo de la obesidad precoz en niños de dos a seis años; el efecto dosis-respuesta entre la duración de la LM y la reducción del riesgo de obesidad se ha estudiado principalmente en la primera infancia, excluyendo a la población adolescente ${ }^{4}$.

Asimismo, factores como la dieta y la actividad física aunados a la duración de la LM, no se han analizado en conjunto como riesgos de la obesidad en niños en edad escolar y adolescentes. El objetivo de este estudio es examinar la asociación entre la duración de la lactancia materna y la alimentación mixta con la obesidad, riesgo cardiometabólico (WHtrR), grasa corporal, conductas de riesgo dietéticas y de actividad física en escolares.

\section{Sujetos y Método}

Tipo de estudio y selección de la población: se realizó un estudio transversal con 1.467 estudiantes de 40 escuelas y 24 colegios (privados y públicos) de Costa Rica. Se utilizó la fórmula de estimación de proporciones con un intervalo de confianza del $95 \%$ y un error 
estándar del 3\%. El cálculo se realizó considerando una varianza máxima $(\mathrm{p}=0,5)$ y el efecto del diseño se estimó en 2. En cada centro educativo, un total de aproximadamente 23 niños y adolescentes fueron seleccionados aleatoriamente utilizando la edad (7 a 15 años), como único criterio de inclusión. Los criterios de exclusión incluyeron información incompleta del participante e incapacidad para leer y escribir.

Se aplicó una guía de entrevistas a los padres para recopilar información sobre la educación y los hábitos del niño, incluida la duración de la LM y la alimentación mixta. También se exploraron variables demográficas y socioeconómicas. Se entrevistó a los niños de primero, segundo y tercer año de escuela.

\section{Lactancia y alimentación infantil}

Los datos sobre la duración de la LM se obtuvieron de las madres o cuidadores. Se les solicitó información retrospectiva, sobre las prácticas de alimentación de los niños. Las 53 madres que respondieron "no estoy seguro" o "prefieren no responder" fueron excluidas del análisis.

La alimentación de los sujetos se definió mediante dos variables: duración de la LM y el tiempo en que se introdujo la fórmula, según las siguientes opciones: a) $<1$ semana, b) $\geq 1$ semana a $<3$ meses, c) $\geq 3$ meses a $<6$ meses, d) $\geq 6$ meses a $<9$ meses, e) $\geq 9$ meses a $<1$ año) y f) $\geq 1$ año. Luego se categorizó la alimentación del participante examinando la respuesta a ambas variables, definiendo "sólo amamantados", "sólo fórmula” y "alimentación mixta”. Los participantes clasificados en la categoría "sólo amamantados" corresponden a aquellos que fueron amamantados durante al menos 6 meses y nunca se les introdujo fórmula. Los clasificados en la categoría "sólo fórmula" incluyó a aquellos que fueron amamantados por menos de 1 semana o solo los alimentaron con fórmula.

Respecto a la alimentación mixta se clasificó de la siguiente manera: a) "Alimentación mixta $\geq 6$ meses" incluyó a los participantes que fueron amamantados durante al menos 6 meses y luego se les introdujo fórmula y b) "Alimentación mixta durante $<6$ meses" incluyó a los participantes que fueron amamantados durante menos de 6 meses y además recibieron fórmula.

\section{Datos antropométricos}

El peso $(\mathrm{kg})$ y la grasa corporal se midieron mediante análisis de bioimpedancia en una báscula Tanita SC-331 S (sin columna), que ha sido recomendada para uso en niños y adolescentes ${ }^{7}$.

El peso corporal se midió con el sujeto descalzo y con ropa ligera. La altura se midió en posición erguida, con los maléolos internos juntos y los talones, glúteos, hombros y región posterior de la cabeza en contacto con el aparato.
Se evaluó el índice de masa corporal de cada participante utilizando los Estándares de Crecimiento Infantil de la $\mathrm{OMS}^{8,9}$ y se clasificó el estado nutricional de acuerdo al percentil obtenido en dos grupos: sin obesidad (IMC < 95) y con obesidad (IMC $\geq 95)$.

La circunferencia abdominal se midió en el punto medio entre la costilla inferior y la parte superior de la cresta ilíaca sobre la piel desnuda. El riesgo cardiometabólico se midió utilizando el indicador cintura/talla $(\mathrm{WHtR} \geq 0,5)^{6}$.

Se realizaron dos medidas para cada participante y se calculó el promedio; una tercera medida se realizó si había diferencia mayor de $0,5 \mathrm{~cm}$. Las mediciones fueron realizadas por profesionales estandarizados.

\section{Calidad de la dieta (DQI)}

Se aplicó una encuesta de frecuencia alimentaria para estudiar los hábitos de consumo de alimentos y bebidas de los participantes. Esta encuesta validada fue utilizada por el equipo de investigación en sujetos de la misma edad ${ }^{10}$ de los participantes y se aplicó de forma individual con la supervisión de dos nutricionistas.

\section{Actividad física}

Se realizó la suma de horas de pantalla (0-24 h/ día), considerando la frecuencia de cuatro comportamientos sedentarios durante los días de semana y los fines de semana: ver televisión, jugar en la computadora o consola, conectarse a internet y hablar diariamente por teléfono u otro medio. Teniendo en cuenta:

(a) el tiempo máximo diario recomendado frente a la pantalla $(2 \mathrm{~h})^{11-13}$.

(b) la frecuencia semanal de actividad física fuera de la escuela/colegio (media $\geq 60$ minutos / día: 0: nunca, 7: diaria $)^{14,15}$ y la práctica de actividad física en el centro educativo.

Los sujetos fueron clasificados como "activos" si cumplían con las siguientes dos condiciones: la suma de las horas de pantalla menos de $2 \mathrm{~h}$ por día y la frecuencia semanal de actividad física fuera de la escuela / colegio (promedio $\geq 60$ minutos / día) más de 3 días a la semana. El resto de los participantes fueron clasificados como sedentarios.

\section{Datos sociodemográficos (covariables)}

Se determinó el sexo y la edad; el nivel socioeconómico de los estudiantes mediante la construcción de un índice ${ }^{16}$ considerando la posesión de servicios y bienes materiales en el hogar.

\section{Procedimientos generales y éticos}

Los participantes dieron su consentimiento informado o asentimiento por escrito, aprobados por el Comité Científico Ético del INCIENSA (IC 2010-05). 


\section{Análisis estadístico}

Se utilizaron estadísticos descriptivos para caracterizar a la población por estado nutricional (sin obesidad y con obesidad) y se calcularon las proporciones para las variables categóricas. Se utilizó la prueba de Kolmogorov-Smirnov para verificar que las variables no se desviaron significativamente del comportamiento normal. Las diferencias entre los grupos se explicaron mediante pruebas paramétricas o no paramétricas según la distribución normal. Los datos definidos por más de dos categorías se probaron mediante ANOVA o la prueba de Kruskal-Wallis según la distribución. Se aplicaron modelos logísticos para organizar el agrupamiento de las observaciones de los estudiantes mediante regresiones univariadas y multivariables. En la regresión univariante se evalúo la asociación entre la duración de la LM y el estado nutricional, así como la asociación entre la variable de alimentación mixta y el estado de nutricional. En los análisis multivariables, las asociaciones se ajustaron por género, ingresos del hogar y educación de los padres. Posteriormente para obtener un modelo integral y final, se ajustaron por el DQI (saludable $=0$ y no saludable $=1$ ), la actividad física ( $(\mathrm{kg})$ y el WHtrR $(\leq 0,5=0,>0,5=1)$. Razones de probabilidades (OR) y los IC del 95\% se calcularon por separado ( $\sin$ obesidad y con obesidad). Para ejecutar los modelos, el método elegido fue "backwards", por lo que el contraste para la eliminación se basó en la probabilidad del estadístico de Wald.

Los análisis fueron realizados en el programa SPSS para Windows (versión 22.0) y se consideró estadísticamente significativo un valor de $\mathrm{p} \leq 0,05$.

\section{Resultados}

La edad media de la población de estudio fue de 11,44 $\pm 2,64$ años; la mayoría de clase media y sedentaria y 50,9\% eran hombres; una mayor proporción de niños entre 7 y 9 años presentó obesidad ( $\mathrm{p}<0,002)$. Las tres cuartas partes de los sujetos con obesidad recibieron leche materna por menos de 6 meses, mientras que una proporción similar de los que no presentaron obesidad fueron amamantados por 6 meses o más $(75,7 \%$ vs $25,9 \%, \mathrm{p}<0,001) \mathrm{y}$, aproximadamente una cuarta de los participantes sin obesidad nunca recibió fórmula (tabla 1).

Se encontraron diferencias estadísticamente significativas con la introducción temprana de fórmula ( 0 - 6 meses de edad) (10,6\% sin obesidad vs. $31,1 \%$, con obesidad, $\mathrm{p}<0,001)$ y con la inclusión de esta a los 6 meses o más, entre los niños sin obesidad $(65,7 \%)$ y con obesidad $(31,7 \%)(\mathrm{p}<0,001)$ (tabla 1$)$.

Proporciones más altas de niños con obesidad en comparación con niños sin obesidad tenían WHtrR con riesgo ( $\mathrm{p}<0,001)$, DQI no saludable $(\mathrm{p}<0,001)$, padres añosos $(\mathrm{p}<0,011)$ y padres con estudios universitarios completos ( $\mathrm{p}<0,001)$ (tabla 1$)$.

En la tabla 2 se observa que los niños y adolescentes que fueron alimentados solo con leche materna $(\mathrm{p}<0,001)$ o en combinación con fórmula durante 6 meses o más $(\mathrm{p}<0,001)$ tuvieron menos probabilidad de obesidad; mientras que los que solo fueron alimentados con fórmula, presentaron más probabilidad de obesidad en comparación con los que no tenían obesidad ( 60,8 vs. 39,2 ; p < 0,005). No se encontraron diferencias estadísticamente significativas entre niños y adolescentes independientemente del estado nutricional respecto a la alimentación mixta por menos de 6 meses $(59,0 \%$ vs. $41,0 \%, \mathrm{p}=0,11)$.

En la tabla 3 se observan los promedios de masa grasa total; al comparar solo LM versus alimentación mixta durante $\geq 6$ meses, no se encontraron asociaciones estadísticamente significativas. No obstante, al comparar solo la LM versus la alimentación mixta durante $<6$ meses, se encontraron asociaciones estadísticamente significativas en los tres grupos de edad analizados. Los niños y adolescentes menos "grasosos" fueron los alimentados únicamente con leche materna. En relación con los promedios de la masa grasa total, comparando los niños solo amamantados con los alimentados solo con fórmula, se encontraron asociaciones estadísticamente significativas en niñas y niños de los tres grupos de edad (excepto en niños de 10 a 12 años).

Se ajustaron las covariables sociodemográficas de los estudiantes y sus padres para determinar las asociaciones (OR) entre los cuatro grupos de duración de la LM examinados y el estado nutricional ( $\sin$ obesidad $=0$ y con obesidad $=1$ ) de los de niños y adolescentes participantes, comparándolos con aquellos que fueron amamantados por menos de una semana o nunca. Las razones de chances que indican mayor protección de la lactancia respecto a la probabilidad de ser obeso se encontraron en los períodos de duración de LM, de una semana a menos de 3 meses y de 9 meses o más. No se encontró asociación entre los niños y adolescentes que fueron amamantados de seis meses a menos de 9 meses (tabla 4). Las asociaciones (OR) para los cuatro grupos de duración de LM examinados respecto a la probabilidad de ser obeso, fueron significativas en el modelo totalmente ajustado con variables sociodemográficas, WHtrR, grasa corporal, DQI y estado de actividad física. En todas las categorías evaluadas de duración de la lactancia materna y alimentación mixta, los niños y adolescentes fueron significativamente menos propensos a ser obesos, en comparación con aquellos que fueron amamantados por menos de una semana o nunca. A pesar del ajuste total, las razones de 
Tabla 1. Características de la población de estudio según estado nutricional

\begin{tabular}{|c|c|c|c|c|}
\hline \multirow[t]{2}{*}{ Variables } & \multirow{2}{*}{$\begin{array}{c}\text { Total } \\
\mathrm{n}=1.467(100 \%)\end{array}$} & \multicolumn{2}{|c|}{ Estado nutricional } & \multirow[t]{2}{*}{$p^{*}$} \\
\hline & & $\begin{array}{c}\text { Sin obesidad } \\
n=1.233(84 \%)\end{array}$ & $\begin{array}{c}\text { Con obesidad } \\
n=234(16 \%)\end{array}$ & \\
\hline \multicolumn{5}{|l|}{ Sexo } \\
\hline Femenino & 49,1 & 48,2 & 53,8 & 0,29 \\
\hline Masculino & 50,9 & 51,8 & 46,2 & 0,33 \\
\hline \multicolumn{5}{|l|}{ Grupos de edad (años) } \\
\hline $7-9$ & 24,7 & 21,8 & 39,3 & 0,002 \\
\hline $10-12$ & 36,3 & 38 & 27,4 & 0,13 \\
\hline $13-15$ & 39,0 & 40,1 & 33,3 & 0,30 \\
\hline \multicolumn{5}{|l|}{ Duración de la lactancia } \\
\hline$<1$ semana & 13,2 & 6,2 & 50,4 & $<0,001$ \\
\hline 1 semana $-<3$ meses & 5,6 & 3,8 & 15,0 & 0,16 \\
\hline $3-<6$ meses & 14,9 & 15,9 & 10,3 & 0,67 \\
\hline $6-<9$ meses & 59,9 & 68,0 & 17,1 & $<0,001$ \\
\hline 9 meses $-<1$ año & 3,2 & 2,8 & 5,1 & 0,58 \\
\hline$\geq 1$ año & 3,1 & 3,3 & 2,1 & 0,22 \\
\hline \multicolumn{5}{|l|}{ Introducción de la fórmula } \\
\hline Nunca & 20,5 & 23,8 & 3,4 & 0,35 \\
\hline$<1$ semana & 6,13 & 0,5 & 3,8 & 0,47 \\
\hline 1 semana - 3 meses & 9,3 & 8,6 & 12,8 & 0,73 \\
\hline $3-<6$ meses & 3,5 & 1,5 & 14,5 & 0,31 \\
\hline $6-<9$ meses & 14,6 & 15,3 & 10,7 & 0,75 \\
\hline 9 meses $-<1$ año & 40,3 & 44,4 & 18,4 & 0,002 \\
\hline$\geq 1$ año & 5,5 & 6,0 & 2,6 & 0,57 \\
\hline \multicolumn{5}{|c|}{ Riesgo cardio-metabólico (relación cintura-talla) (WHtrR) } \\
\hline Sin riesgo & 79,9 & 90,4 & 24,4 & $<0,001$ \\
\hline Con riesgo & 20,1 & 19,6 & 75,6 & $<0,001$ \\
\hline \multicolumn{5}{|l|}{ Calidad de la dieta } \\
\hline Saludable & 11,3 & 9,7 & 11,5 & 0,94 \\
\hline No saludable & 88,7 & 76,6 & 88,5 & $<0,001$ \\
\hline \multicolumn{5}{|l|}{ Actividad física } \\
\hline Activo & 40,5 & 41,4 & 35,9 & 0,40 \\
\hline Sedentario & 59,5 & 58,6 & 64,1 & 0,24 \\
\hline
\end{tabular}

$p^{*}$ : estadísticamente significativo si $p \leq 0,05 ; n=$ número de niños y adolescentes. WHtrR: riesgo cardiomatabólico.

Tabla 2. Tipo de alimentación según estado nutricional de la población de estudio

\begin{tabular}{|c|c|c|c|c|}
\hline \multirow[t]{2}{*}{ Tipo de alimentación } & \multirow{2}{*}{$\begin{array}{c}\text { Total } \\
n=1.467(100 \%)\end{array}$} & \multicolumn{2}{|c|}{ Estado nutricional } & \multirow[t]{2}{*}{$p^{*}$} \\
\hline & & $\begin{array}{c}\text { Sin obesidad } \\
n=1.233(84 \%)\end{array}$ & $\begin{array}{l}\text { Con obesidad } \\
n=234(16 \%)\end{array}$ & \\
\hline Solo amamantado & 20,5 & 97,3 & 2,7 & $<0,001$ \\
\hline Alimentación mixta durante $\geq 6$ meses & 59,4 & 92,3 & 7,7 & $<0,001$ \\
\hline Alimentación mixta durante $<6$ meses & 6,8 & 59,0 & 41,0 & 0,11 \\
\hline Únicamente fórmula & 13,2 & 39,2 & 60,8 & 0,005 \\
\hline
\end{tabular}

$p^{*}$ : estadísticamente significativo si $p \leq 0,05 ; n=$ número de niños y adolescentes. 
Tabla 3. Comparación de la masa grasa total media ${ }^{a}(\mathbf{k g})^{\mathrm{b}}$ en niños y adolescentes que solo fueron amamantados respecto a los que recibieron alimentación mixta o solo fórmula

\begin{tabular}{|c|c|c|c|c|}
\hline Población & Solo amamantado & $\begin{array}{l}\text { Alimentación mixta } \\
\text { durante } \geq 6 \text { meses }\end{array}$ & $\begin{array}{l}\text { Alimentación mixta } \\
\text { durante }<6 \text { meses }\end{array}$ & Solo fórmula \\
\hline \multicolumn{5}{|l|}{ Femenino } \\
\hline $7-9$ años $(n=179)$ & $5,63(3,60)$ & $5,76(3,32)$ & $12,10(4,56)^{\star}$ & $9,38(3,77)^{*}$ \\
\hline 10-12 años $(n=261)$ & $7,26(2,96)$ & $7,37(3,48)$ & $9,87(4,23)^{*}$ & $8,32(4,16)$ \\
\hline $13-15$ años $(n=281)$ & $10,51(4,21)$ & $11,51(4,22)$ & $13,78(2,58)^{*}$ & $18,58(8,59)$ * \\
\hline \multicolumn{5}{|l|}{ Masculino } \\
\hline $7-9$ años $(n=182)$ & $4,92(2,77)$ & $5,03(2,46)$ & $7,77(4,36)^{*}$ & $7,51(4,63)^{*}$ \\
\hline $10-12$ años $(n=272)$ & $5,24(2,23)$ & $5,64(3,60)$ & $9,33(6,03)^{*}$ & $5,31(3,15)$ \\
\hline $13-15$ años $(n=292)$ & $6,74(3,95)$ & $6,82(4,62)$ & $12,3(6,30)^{*}$ & $17,11(7,54)^{*}$ \\
\hline
\end{tabular}

aTodos los valores son promedios y desviación estándar en paréntesis. ${ }^{b}$ Medido mediante bioimpedancia en una Tanita SC-331 S (sin columna). ${ }^{*}$ Indica que $\mathrm{p}$ valor $<0,05$ (Prueba de Kruskal-Wallis).

\begin{tabular}{|c|c|c|c|}
\hline $\begin{array}{l}\text { Duración de la lactancia materna / } \\
\text { Alimentación mixta }\end{array}$ & $\begin{array}{l}\text { OR no ajustado } \\
\text { (IC del } 95 \%)\end{array}$ & $\begin{array}{l}\text { OR ajustado por variables } \\
\text { sociodemográficas de } \\
\text { estudiantes y sus padres }\end{array}$ & $\begin{array}{l}\text { OR ajustado por variables socio- } \\
\text { demográficas, grasa corporal (Kg), DQI, } \\
\text { estado de actividad física y WHtrR }\end{array}$ \\
\hline$<1$ semana & 1 & 1 & 1 \\
\hline 1 semana $-<3$ meses & $0,71(0,69-0,74)^{*}$ & $0,70(0,67-0,73)$ * & $0,74(0,72-0,81)$ * \\
\hline $3-<6$ meses & $0,83(0,80-0,86)^{*}$ & $0,92(0,88-0,96)$ * & $0,81(0,77-0,86)$ * \\
\hline $6-<9$ meses & $1,06(1,02-1,09) *$ & $0,95(0,99-1,03)$ & $0,95(0,90-0,99)$ * \\
\hline 9 meses $-<1$ año & $0,74(0,71-0,77)^{\star}$ & $0,73(0,70-0,76)$ * & $0,69(0,64-0,74)$ * \\
\hline$\geq 1$ año & $0,76(0,73-0,79)^{*}$ & $0,76(0,73-0,79)$ * & $0,77(0,72-0,82)$ * \\
\hline Solo amamantado & 1 & 1 & 1 \\
\hline Alimentación mixta durante $\geq 6$ meses & $7,12(5,88-11,78)^{\star *}$ & $7,00(5,22-10,81)$ * & $7,09(5,24-10,89)$ * \\
\hline Alimentación mixta durante $<6$ meses & $2,19(1,30-3,71)^{\star}$ & $2,16(1,21-3,60)$ * & $2,18(1,22-3,69)$ * \\
\hline Solo fórmula & $18,86(13,50-26,61)^{*}$ & $17,46(13,10-25.83)$ * & $18,80(13,20-26,00)$ * \\
\hline \multicolumn{4}{|c|}{$\begin{array}{l}\text { WHtrR: riesgo cardiomatabólico. *Indica que } \mathrm{p} \text { valor }<0,05 \text { (prueba de Wald). }{ }^{\mathrm{a} A j u s t a d o ~ p o r ~ g e ́ n e r o ~}(1=\text { femenino y } 0=\text { masculino) y edad } \\
\text { (años, variable de continuación) del niño/adolescente; nivel socioeconómico familiar (índice, variable continuar) y educación de los padres } \\
\text { (años de estudio, variable continuar). }{ }^{\text {B}} \mathrm{Ajustado} \text { por género }(1=\text { femenino y } 0=\text { masculino), edad (años, variable continuar), WHtrR }(1=>0,50 \\
\text { y } 0=\leq 0,50) \text {, grasa corporal (Kg, variable continua), DQI ( } 1=\text { no saludable y } 0=\text { saludable) y estado de actividad física }(1=\text { sedentario y } \\
0=\text { activo) del niño/adolescente; nivel socioeconómico familiar (índice, variable continua) y educación de los padres (años de estudio, variable } \\
\text { continua). }\end{array}$} \\
\hline
\end{tabular}

chances de mayor protección de la lactancia respecto a la probabilidad de ser obeso continuaron siendo las comprendidas en los períodos de duración de LM, de una semana a menos de 3 meses y de 9 meses o más.

En los tres modelos realizados, los niños y adolescentes que tuvieron más probabilidades de ser obesos en comparación con los que solo recibieron LM, fueron aquellos que solo fueron alimentados con fórmula, seguidos por los que recibieron alimentación mixta por 6 o más meses (tabla 4).

\section{Discusión}

Considerando la OR obtenida en el modelo totalmente ajustado, donde se incluyó entre otros factores, el riesgo cardiovascular, para analizar la asociación entre la duración de la LM y la probabilidad de obesidad, se observó que cualquier tiempo superior a una semana protege al niño y al adolescente de la obesidad. Por la ausencia en la literatura científica de estudios que hayan analizado esos factores y reportado razones de 
chances durante ese período específico de duración de LM, no es posible comparar nuestros resultados con otros. Una posible explicación es que la adición de fórmula, ya sea como alimentación exclusiva o suplemento, conlleva al aumento de peso no fisiológico que se asocia con la obesidad ${ }^{17}$. Además, se recomienda iniciar la alimentación complementaria posterior a las 17 semanas de edad ${ }^{18}$.

Poorolajal et $\mathrm{al}^{19}$ reportaron una OR (IC del 95\%) para la $\mathrm{LM}<4$ meses frente $\mathrm{a} \geq 4$ meses de $1,24^{1,16,1,33}$, lo que significa que la $\mathrm{LM}<4$ meses puede aumentar el riesgo de obesidad infantil en un $24 \%(\mathrm{p}=0,001)$. La tendencia de duración de LM y su protección de la obesidad de nuestro estudio es respaldado por Rito et $\mathrm{a}^{20}$, respecto a que, cuanto más exclusivamente y por más tiempo se amamanta a los niños, mayor es la protección frente a la obesidad. No obstante, en nuestro estudio, en los períodos de duración de LM examinados de 3 a 9 meses, la protección de LM respecto al riesgo de obesidad fueron menores comparados con el resto de períodos; esta variación podría estar explicada por el diferente número de participantes en cada uno de los grupos de duración de LM examinados. A pesar de esto, Spatz ${ }^{21}$ reporta que, cualquier tiempo de amamantamiento ayudará a proteger al niño de la obesidad.

Aunque es deseable que los niños sean alimentados sin fórmula, algunas madres de este estudio lo hicieron, práctica que se asocia con la obesidad. Son varios los mecanismos que podrían explicar esta asociación ${ }^{21,22}$ : los niños que son amamantados tienen una menor ingesta de proteínas y, en general, consumen menos calorías; la alimentación con fórmula conduce a una mayor respuesta de la insulina, lo que estimula a la deposición de tejido graso y por ende, al riesgo cardiometabólico, obesidad y diabetes tipo 2 .

La LM de manera exclusiva está inversamente asociada con la velocidad del aumento de peso durante el primer año de vida ${ }^{23}$; los niños amamantados autorregulan la ingesta, independientemente de la disponibilidad de leche en el pecho; por lo que dejará de alimentarse cuando esté satisfecho, mientras que, los niños alimentados con biberón tienen una autorregulación deficiente y un mayor aumento de peso al final de la infancia ${ }^{21}$.

No obstante, nuestros resultados sugieren que la alimentación mixta es menos riesgosa que si sólo se alimenta con fórmula ( $\mathrm{OR}=18,8$, IC 95\% 13,2-26,0). Los niños amamantados consumen inicialmente volúmenes pequeños de calostro, lo que les permite que utilicen la grasa parda destinada a sustentarlos y la digestión de grasas de la leche materna es superior a la de la fórmula por las lipasas que posee $e^{21,24}$.

La duración de la LM se asocia con elecciones de alimentos más saludables desde la primera infancia ${ }^{24-26}$; las preferencias alimentarias de los niños parecen estar determinadas por la exposición a los alimentos y sabores de la dieta de la madre, a través del líquido amniótico y la leche materna ${ }^{25}$. Trabulsi et $\mathrm{al}^{27}$ reportan que cada día que un niño se amamanta, su paladar y las papilas gustativas están expuestos a nuevos sabores, mientras que la fórmula siempre sabe igual. Una investigación realizada en Holanda encontró que los niños de 7 años amamantados durante más de 16 semanas tenían una mayor ingesta de frutas y vegetales y menos probabilidades de consumir pan blanco, refrescos, barras de chocolate y alimentos fritos que los que nunca fueron amamantados ${ }^{28}$.

Respecto a la introducción de alimentos sólidos y otros líquidos diferentes a la leche materna y a la fórmula, analizados en este estudio transversal y con sesgo de memoria ${ }^{29}$, es atrevido sugerir que los niños que fueron alimentados por menos de 6 meses de manera mixta versus los solo amamantados, tuvieron OR menores (menos riesgo) que aquellos que fueron alimentados de manera mixta por más de 6 meses, debido a que los primeros se expusieron menos a la fórmula.

Por esas razones, en el momento de la introducción de alimentos sólidos, los niños que hayan recibido únicamente leche materna o hayan sido alimentados de manera mixta, pero con predominancia de leche humana, habrán estado expuestos a tener más experiencias respecto a los gustos de los alimentos en comparación con sus pares no amamantados o alimentados de manera mixta, pero predominantemente con fórmula.

Por ello, se recomienda adherirse a las guías alimentarias desde la primera infancia para promover la construcción de hábitos alimentarios saludables en los niños y prevenir el desarrollo de conductas de riesgo y, en consecuencia, la obesidad ${ }^{30}$.

El mismo patrón aparece con la asociación de la duración de la LM por menos de 6 meses y el riesgo para la salud, que combina una alimentación poco saludable y un estilo de vida sedentario. La evidencia señala que comer, mientras se ve la televisión se asocia con una baja calidad de dieta y obesidad, debido al consumo frecuente de bebidas azucaradas y alimentos ricos en grasas y azúcares y menos frutas y vegetales ${ }^{31}$. Estudios han reportado que ver televisión durante más de $2 \mathrm{~h} /$ día puede aumentar el riesgo de obesidad en un $42 \%(\mathrm{p}=0,001)^{19}$ y que, la LM muestra efectos beneficiosos sobre la aptitud física de los niños ${ }^{32}$. En países en desarrollo se ha evidenciado que la duración de la LM se asocia a un mayor aumento en la aptitud cardiorrespiratoria en la infancia ${ }^{33}$. Otros estudios han reportado que la LM en la primera infancia influye positivamente en las habilidades para caminar durante la niñez y años posteriores, lo cual podría explicarse por los ingredientes activos, enzimas y adipocinas presentes en la leche materna ${ }^{34}$. Los niveles de factor de crecimiento 
similar a la insulina tipo 1 (IGF-1) podrían estar desempeñando un papel fundamental en la musculatura y en la fuerza en los niños amamantados ${ }^{35}$, pues estos niveles de IGF-1 se asocian positivamente con la ingesta de energía y de proteína neonatal y con el crecimiento muscular y óseo ${ }^{36,37}$. La ingesta de proteínas en la primera infancia puede contribuir a programar la masa magra y el IGF-I alrededor de la pubertad ${ }^{37-40}$.

La dieta de baja calidad y el sedentarismo combinados son ampliamente reconocidos como sinérgicos para aumentar el riesgo de obesidad y, en consecuencia, el desarrollo de ECNT ${ }^{41}$. Singhal y Lucas ${ }^{42}$ plantearon la "hipótesis de la aceleración del crecimiento", la cual postula que una tasa acelerada de aumento de peso, estatura y adiposidad durante períodos críticos, tal como la infancia, puede programar características metabólicas adversas y riesgo de enfermedad cardiovascular. En nuestro estudio, los niños con riesgo cardiometabólico, dieta no saludable y sedentarismo, alimentados solo con fórmula tuvieron altas probabilidades de obesidad. Finalmente, en el modelo totalmente ajustado (tabla 4), se observa que la LM se mantuvo como factor protector de la obesidad, resultados que son consistentes con otros estudios ${ }^{43,44}$.

Este estudio evidencia el poder protector de la LM en períodos de duración que no se habían examinado (desde una semana a más de un año de edad), respecto al riesgo de obesidad en niños y adolescentes. Los resultados son útiles para implementar políticas para la promoción de la salud pública y la prevención de la obesidad y las ECNT.

Costa Rica y los países del mundo deben apoyar el inicio de la LM desde el nacimiento y su larga duración, en línea con la Iniciativa de hospitales y centros de salud amigos del niño; la disponibilidad de espacios acogedores para la LM y las campañas educativas, que apoyan a las mujeres en su decisión de amaman$\operatorname{tar}^{24,45,46}$. Asimismo, se deben fortalecer los Bancos de Leche Humana, las Clínicas de LM y Desarrollo y las redes de apoyo materno infantil, integradas en una cultura de LM positiva ${ }^{24,47}$.

Por último, entre las limitaciones de este estudio está el uso de la bioimpedancia, ya que no es un método considerado estándar de oro para medir la com- posición corporal y el posible sesgo de memoria en las madres y padres de familia; los datos sobre la LM y alimentación mixta de los participantes podrían no ser exactos, pues se remontan de 7 a 15 años al momento de la entrevista. Es un estudio transversal no diseñado para evaluar las asociaciones entre LM y conductas de riesgo para la salud o sobrepeso / obesidad; no se contó con información sobre los primeros hábitos de alimentación y actividad física de los participantes durante la primera infancia, lo que sería beneficioso para explicar el papel de la crianza y los estilos de vida familiares en el desarrollo de la obesidad.

Estos resultados deben alentar a los profesionales de la salud a brindar apoyo y asesoramiento a las madres y cuidadores de niños y adolescentes, sobre la importancia de la alimentación complementaria, las pautas de alimentación saludable y la actividad física durante las primeras etapas de la vida, para frenar el aumento de obesidad ${ }^{41}$.

\section{Responsabilidades Éticas}

Protección de personas y animales: Las autoras declaran que los procedimientos seguidos se conformaron a las normas éticas del comité de experimentación humana responsable y de acuerdo con la Asociación Médica Mundial y la Declaración de Helsinki.

Confidencialidad de los datos: Las autoras declaran que han seguido los protocolos de su centro de trabajo sobre la publicación de datos de pacientes.

Derecho a la privacidad y consentimiento informado: Las autoras han obtenido el consentimiento informado de los pacientes y/o sujetos referidos en el artículo. Este documento obra en poder de la autora de correspondencia.

\section{Conflicto de intereses}

Las autoras declaran no tener conflicto de intereses.

\section{Referencias}

1. Berman I, Ortiz O, Pineda L, et al. Los primeros mil días de vida. Una mirada rápida. An Med Asoc Med Hosp ABC. 2016;61(4):313-8. Disponible en: http://www.medigraphic.com/ analesmedicos.

2. Horta B, Loret de Mola C, Victora C. Long-term consequences of breastfeeding on cholesterol, obesity, systolic blood pressure and type 2 diabetes: a systematic review and meta-analysis. Acta Paediatr. 2015;104(467):30-7. doi: 10.1111/ apa.13133.

3. Specht IO, Rohde JF, Olsen NJ, et al. Duration of exclusive breastfeeding may be related to eating behaviour and dietary intake in obesity prone normal weight young children. PLoS
ONE 2018;13(7):e0200388. https://doi. org/10.1371/ journal.pone.0200388.

4. Qiao J, Dai L-J, Zhang Q, et al. A Meta-Analysis of the Association Between Breastfeeding and Early Childhood Obesity. J Pediatr Nurs. 2020;53:57-66. https://doi.org/10.1016/j. pedn.2020.04.024.

5. Gupta A, Suri S, Dadhich J, et al. The World Breastfeeding Trends Initiative: 
Implementation of the Global Strategy for Infant and Young Child Feeding in 84 countries. J Public Health Policy. 2019;40(1):35-65. doi: 10.1057/s41271018-0153-9. PMID: 30538269.

6. Browning L, Hsieh S, Ashwell M. A systematic review of waist-to-height ratio as a screening tool for the prediction of cardiovascular disease and diabetes: 0.5 could be a suitable global boundary value. Nutr. Res. Rev. 2010;23:247-69. doi: 10.1017/ S0954422410000144.

7. Tanita Corporation. Body composition analyzer SC-331S. Instruction Manual. Estados Unidos: Tanita Corporation 2013.

8. de Onis M, Onyango A, Borghi E. WHO Child Growth Standards Length/heightfor-age, weight-for-age, weight-forlength, weight-for-height and body mass index-for age. Methods and development World Health Organization. 2009 (Web page] http://www.who.int/childgrowth/ standards/Technical_report.pdf?ua=1.doi: 10.1080/08035320500495548.

9. Güngör N. Overweight and obesity in children and adolescents. J Clin Res Pediatr Endocrinol. 2014;6(3):129-43. doi:10.4274/jcrpe.1471.

10. Núñez H, Holst I, Campos N. New Diet Quality Index for children and adolescents in Costa Rica. Nutr Hosp. 2020;37(1):65-72. doi: http://dx.doi. org/10.20960/nh.02695)

11. American Academy of Pediatrics. Children, Adolescents, and Television. Pediatrics 2001:107(2):423-6. doi: 10.1542/peds.107.2.423.

12. Reid Y, Radesky J, Christakis D, et al. APP COUNCIL ON COMMUNICATIONS AND MEDIA. Children and Adolescents and Digital Media (Review). Pediatrics. 2016;138(5):e20162593. doi: 10.1542/ peds.2016-2593.

13. Tremblay M, Carson V, Chaput J. et al. Canadian 24-hour movement guidelines for children and youth: an integration of physical activity, sedentary behaviour, and sleep. Appl Physiol Nutr Metab. 2016;41(6 Suppl 3):S311-27. doi: 10.1139 / apnm-2016-0151.

14. U.S. Department of Health and Human Services. Physical Activity Guidelines for Americans and Youth Physical Activity Guidelines Toolkit. Author, Washington DC. 2008. http://www.health.gov/ PAguidelines/guidelines/chapter3.aspx.

15. Hyde E, Omura J, Watson K, et al. Knowledge of the Adult and Youth 2008 Physical Activity Guidelines for Americans. J Phys Act Health. 2019;16(8):618-22. doi: 10.1123/ jpah.2018-0143.

16. Madrigal J. La construcción de índices. Editorial Universidad de Costa Rica, San José 1997.

17. Feldman-Winter L, Kellams A, Peter-
Wohl S, et al. Evidence-Based Updates on the First Week of Exclusive Breastfeeding Among Infants $\geq 35$ Weeks. Pediatrics. 2020;145(4):e20183696. doi: 10.1111/ mcn.12472.

18. Fabiano V, Albani E, Cammi GM, Zuccotti GV. Nutrition in developmental age: few rules to stay healthy. Minerva Pediatr. 2020;72(3):182-95. https://doi. org/10.23736/S0026-4946.20.05803-X

19. Poorolajal J, Sahraei F, Mohamdadi Y, Doosti-Irani A, Moradi L. Behavioral factors influencing childhood obesity: a systematic review and meta-analysis. Obes Res Clin Pract. 2020;14(2):109-18. https:// doi.org/10.1016/j.orcp.2020.03.002.

20. Rito AI, Buoncristiano M, Spinelli A, et al. Association between Characteristics at Birth, Breastfeeding and Obesity in 22 Countries: The WHO European Childhood Obesity Surveillance Initiative-COSI 2015/2017. Obes Facts. 2019;12(2):226-43. doi: $10.1159 / 000500425$.

21. Spatz DL. Preventing obesity starts with breastfeeding. J Perinat Neonatal Nurs. 2014;28(1):41-50. doi: 10.1097/ JPN.0000000000000009.

22. Gridneva Z, Rea A, Hepworth A, et al. Relationships between Breastfeeding Patterns and Maternal and Infant Body Composition over the First 12 Months of Lactation. Nutrients. 2018;5;10(1):45. doi: 10.3390/nu10010045.

23. Azad M, Vehling L, Chan D, et al. Infant Feeding and Weight Gain: Separating Breast Milk From Breastfeeding and Formula From Food. Pediatrics. 2018;142(4):e20181092. doi: 10.1542/ peds.2018-1092.

24. Harrison M, Brodribb W, Hepworth JA. Qualitative systematic review of maternal infant feeding practices in transitioning from milk feeds to family foods. (Review). Matern Child Nutr. 2017;13(2):e12360. doi: $10.1111 / \mathrm{mcn} .12360$.

25. Ventura A. Does Breastfeeding Shape Food Preferences? Links to Obesity. Ann Nutr Metab. 2017;70(Suppl 3):8-15. doi: $10.1159 / 000478757$.

26. Murray R. Influences on the initial dietary pattern among children from birth to 24 months. Nutrition Today. 2017;52 (Issue2):S25-S29. doi: 10.1097/ NT.0000000000000195

27. Trabulsi JC, Mennella JA. Diet, sensitive periods in flavour learning, and growth. Int Rev Psychiatry. 24(3):219-30. doi:10.3 109/09540261.2012.675573.

28. Scholtens S, Brunekreef B, Smit HA, et al. Do Differences in Childhood Diet Explain the Reduced Overweight Risk in Breastfed Children? Obesity. 2008;16(11):2498-503. doi:10.1038/oby.2008.403.

29. Manterola C, Quiroz G, Salazar P, et al. Metodología de los tipos y diseños de estudio más frecuentemente utilizados en investigación clínica. Rev. Med. Clin. Condes 2019;30(1):36-49. doi. org/10.1016/j.rmclc.2018.11.005)

30. Batista M, Žigić L, Žaja O, et al. Predictors of eating disorder risk in anorexia nervosa adolescents. Acta Clin Croat. 2018;57(3):399-410. doi: 10.20471/ acc.2018.57.03.01.

31. Avery A, Anderson C, McCullough F. Associations between children's diet quality and watching television during meal or snack consumption: A systematic review. Matern Child Nutr. 2017;13(4):e12428. doi: 10.1111/ mcn. 12428

32. Heshmati J, Sepidarkish M, Shidfar F, et al. Effect of Breastfeeding in Early Life on Cardiorespiratory and Physical Fitness: A Systematic Review and Meta-Analysis. Breastfeed Med Off J Acad Breastfeed Med. 2018;13(4):248-58. doi.org/10.1089/ bfm.2018.0001.

33. Vafa M, Heshmati J, Sadeghi H, et al. Is exclusive breastfeeding and its duration related to cardio respiratory fitness in childhood? J Matern-Fetal Neonatal Med Off J Eur Assoc Perinat Med Fed Asia Ocean Perinat Soc Int Soc Perinat Obstet. 2016;29(3):461-5. https://doi.org/10.3109/ 14767058.2015.1004052.

34. Savino F, Liguori SA, Lupica MM. Adipokines in breast milk and preterm infants. Early Hum Dev. 2010;86(Suppl 1):77-80. https://doi.org/10.1016/j. earlhumdev.2010.01.011.

35. Martin RM, Holly JMP, Smith GD, et al. Could associations between breastfeeding and insulin-like growth factors underlie associations of breastfeeding with adult chronic disease? The Avon Longitudinal Study of Parents and Children. Clinical Endocrinology. 2005;62(6):728-37. doi:10.1111/j.1365-2265.2005.02287.x

36. Mazzocchi A, Giannì ML, Morniroli D, et al. Hormones in Breast Milk and Effect on Infants' Growth: A Systematic Review. Nutrients. 2019;11(8):1845. doi:10.3390/ nu11081845.

37. Madsen AL, Larnkjær A, Mølgaard C, et al. IGF-I and IGFBP-3 in healthy 9 month old infants from the SKOT cohort: breastfeeding, diet, and later obesity. Growth Horm IGF Res. 2011;21(4):199204. doi: 10.1016/j.ghir.2011.05.003. Epub 2011 May 31. PMID: 21624842.

38. Switkowski KM, Jacques PF, Must A, et al. Associations of protein intake in early childhood with body composition, height, and insulin-like growth factor I in mid-childhood and early adolescence. Am J Clin Nutr. 2019;109(4):1154-63. doi: 10.1093 / ajcn / nqy354.

39. Lönnerdal B. Infant formula and infant nutrition: bioactive proteins of human milk and implications for 
composition of infant formulas. Am J Clin Nutr. 2014;99(3):712S-doi: 10.3945 / ajen.113.071993.

40. Putet G, Labaune J-M, Mace K, et al. Effect of dietary protein on plasma insulin-like growth factor-1, growth, and body composition in healthy term infants: a randomised, double-blind, controlled trial (Early Protein and Obesity in Childhood (EPOCH) study). Br J Nutr. 2016;115(2):271-84. doi:10.1017/ S0007114515004456.

41. Koletzko B, Godfrey K, Poston L, et al. Early Nutrition Project Systematic Review Group. Nutrition During Pregnancy, Lactation and Early Childhood and its Implications for Maternal and Long-Term Child Health: The Early Nutrition Project Recommendations. (Review). Ann
Nutr Metab. 2019;74(2):93-106. doi: $10.1159 / 000496471$.

42. Singhal A, Lucas A. Early origins of cardiovascular disease: is there a unifying hypothesis? Lancet Lond Engl. 2004;363(9421):1642-5. https://doi. org/10.1016/S0140-6736(04)16210-7

43. Oyarzún M, Barja S, Domínguez M, et al. Breastfeeding, obesity and metabolic syndrome at school age. Rev Chil Pediatr. 2018;89(2):173-81. doi: 10.4067/S037041062018000200173)

44. Lefebvre C, John R. The effect of breastfeeding on childhood overweight and obesity: A systematic review of the literature. J Am Assoc Nurse Pract. 2014;26(7):386-401. doi: 10.1002/23276924.12036.

45. Pérez R, Martínez J, Segura-Pérez S. Impact of the Baby-friendly Hospital
Initiative on breastfeeding and child health outcomes: a systematic review. Matern Child Nutr (Review). 2016;12(3):402-17. doi: 10.1111/ mcn.12294.

46. Canadian Paediatric Society. Position statement: The baby-friendly initiative: Protecting, promoting, and supporting breastfeeding. Retrieved from Canadian Paediatric Society 2012. http://www.cps.ca/en/documents/ position/Baby-friendly-intiativebreastfeeding).

47. Caja Costarricense de Seguro Social. Manual Implementación Clínicas de LM y Desarrollo: Escenario Hospitalario. CCSS: San José, Costa Rica. 2012. https://www. ministeriodesalud.go.cr/gestores_en_ salud/lactancia/CNLM_manual_lactac_ materna_y_desarrollo_2012.pdf. 